\title{
Uso de VANT para Prospecção Eólica em Sistemas Aquáticos: Desenho Amostral e Avanços Instrumentais
}

\author{
Arcilan T. Assireu $^{1}$ (D), José C. Mendonça ${ }^{2}$ (D), Ramon M. Freitas ${ }^{3}$ (D), André L. Reis ${ }^{1}$ (D), \\ Cláudio C. Pellegrini ${ }^{4}$ (D), Felipe M. Pimenta ${ }^{5}$ \\ ${ }^{1}$ Universidade Federal de Itajubá, Itajubá, MG, Brasil. \\ ${ }^{2}$ Universidade Estadual do Norte Fluminense, Campos dos Goytacazes, RJ, Brasil. \\ ${ }^{3}$ Camargo \& Schubert Engenharia do Vento, Curitiba, PR, Brasil. \\ ${ }^{4}$ Universidade Federal de São João Del, São João del Rei, MG, Brasil. \\ ${ }^{5}$ Universidade Federal de Santa Catarina, Florianópolis, SC, Brasil.
}

Recebido em: 25 de Setembro de 2017 - Aceito em: 10 de Dezembro de 2017

\begin{abstract}
Resumo
O Projeto "uso de VANT para prospecção eólica em sistemas aquáticos" consistiu num esforço multi-instrumental e multi-institucional para estudar a camada limite atmosférica influenciada por extensos corpos d'água e pelas feições do entorno como colinas, rampas e mudanças abruptas de rugosidade. Dois trabalhos de campo foram conduzidos em setembro e novembro de 2016 ao longo do sistema aquático e no seu entorno. Durante os experimentos foram utilizadas duas torres anemométricas, uma instalada sobre uma ilha e outra sobre uma colina. Além disso, um sistema flutuante para prospecção eólica, desenvolvido no âmbito do projeto, foi utilizado para hospedar uma torre de $10 \mathrm{~m}$ e um LIDAR Doppler que possibilitou o levantamento de perfis a $200 \mathrm{~m}$, algo inédito em sistemas aquáticos continentais brasileiros. O perfil vertical de temperatura e umidade foi levantado por balões cativos e, um VANT, adaptado para realizar perfis verticais do vento foi utilizado pela primeira vez no Brasil, e é objetivo deste trabalho apresentar os detalhes de seu desenvolvimento bem como da validação dos respectivos dados. Este artigo tem como objetivo também apresentar um visão geral sobre o projeto e descrever a instrumentação e desenho amostral utilizado. Artigos adicionais, submetidos para esta revista, consideram diferentes aspectos da pesquisa e trazem resultados inéditos sobre escoamentos sobre colinas, a influência do fetch nestes sistemas e os aspectos moduladores da estabilidade atmosférica nestes ambientes.
\end{abstract}

Palavras-chave: camada limite planetária, energia eólica, veículo aéreo não tripulado.

\section{Wind Profile in the Aquatic System Using Multirotor Aircraft: Sample Design and Instrumental Advances}

\begin{abstract}
The Project "unmanned aerial vehicles to wind assessment in aquatic systems" was a multi-institutional and multiinstrument experiment carried out to measure, in detail, the spatial characteristics of mean wind over a large tropical aquatic system and how is the flow modulated by hill, ramp and abrupt roughness change. Two field experiments were conducted during September and November 2016 in the Furnas reservoir and surrounding, an extensive $\left(\sim 1,250 \mathrm{~km}^{2}\right)$ Brazilian typical reservoir. During the experiments, two towers were deployed on a hill and isle, and on the anchored buoy was installed a $10 \mathrm{~m}$ mast and a Doppler Lidar with a stabilizing mechanism. Vertical profile of temperature and humidity was obtained by attach sensor to tethered balloon. The present paper provides an overview of the Project as a whole, including a detailed description of the study area and the instrumentation and attempts to prove the viability of using an unmanned 6-motors rotorcraft, commonly known as a hexacopter, as a promising low-cost alternative to traditional platforms for vertical profiling in the atmospheric boundary layer.
\end{abstract}

Keywords: wind energy, planetary boundary layer, unmanned aerial vehicles.

Autor de correspondência: Arcilan T. Assireu, arcilan@unifei.edu.br. 


\section{Introdução}

Este artigo é o primeiro de uma série que trará os resultados das medidas de campo realizadas por dois projetos científicos: "Aproveitamento eólico em reservatórios hidrelétricos" e "Uso de VANT para prospecção eólica em sistemas aquáticos", ambos apoiados pela FAPEMIG. Estes projetos, que envolveram cooperação entre várias instituições como UNIFEI, UFSC, UENF e INPE, representam o maior esforço já realizado no Brasil para estudos da camada limite atmosférica em extensos sistemas aquáticos interiores brasileiros.

O principal objetivo deste projeto é o avanço na compreensão da camada limite atmosférica influenciada por extensos sistemas aquáticos, localizados em regiões de planalto e em condições de entorno marcado por terrenos complexos. Fatores de particular interesse foram a aceleração do escoamento e modificações no regime de turbulência que podem ocorrer na presença de fetch e colinas adjacentes ao sistema aquático. Resultados específicos serão apresentados em alguns artigos submetidos a esta revista e em outros já aceitos. Serão abordados em detalhe as observações do escoamento sobre colinas a partir do perfilhamento com o VANT e modelagem analítica (Assireu et al., 2019.), a influência do fetch do sistema aquático para o escoamento (Nassif et al., submetido a esta Revista), integração dos dados observados com modelagem numérica (Pellegrini et al., 2019), e a influência de mudanças abruptas da rugosidade superficial na interface água terra para o regime de estabilidade atmosférica (Siqueira et al., submetido a esta Revista).

O objetivo do presente artigo é trazer informações gerais sobre o projeto bem como do desenho amostral e instrumentações utilizadas e desenvolvidas no âmbito do projeto, tendo em vista ser uma base para os demais artigos. Especificamente, será detalhado aqui o uso do VANT bem como da validação dos dados, os quais serão utilizados nos vários artigos mencionados acima.

As informações obtidas neste projeto, além de trazerem subsídios para o aproveitamento eólico em reservatórios hidrelétricos e seu entorno, são de interesse também para a dispersão de poluentes atmosféricos, para culturas agrícolas sensíveis ao vento, conforto térmico em construções realizadas no entorno destes sistemas aquáticos e arraste sobre construções. Por fim, os resultados apresentados nos supracitados artigos podem, potencialmente, ser estendidos para regiões litorâneas brasileiras bordejadas por terrenos complexos e que abrigam importantes parques industriais (ex., Baixada Santista) e as usinas nucleares brasileiras (Serra do Mar).

Como vários dos trabalhos citados acima farão uso de dados de um VANT, também é objetivo do presente artigo apresentar os detalhes que envolveram a adaptação deste equipamento para levantamento do perfil do vento, bem como a validação dos dados e potenciais contribuições que este desenvolvimento poderá trazer para a meteorologia. $\mathrm{O}$ uso de aviões tripulados em experimentos de larga escala como complemento a estudos de meteorologia e sensoriamento remoto são bem documentados na literatura, como nos trabalhos de Bange et al. (2002), Beyrich e Mengelkamp (2006), dentre outros. Durante os últimos anos tem havido um grande interesse pelo uso de veículos aéreos não tripulados (VANTs) para estudos em várias áreas do conhecimento. Tradicionalmente vem se utilizando VANTs de asa fixa e, particularmente para estudos de parâmetros meteorológicos pode-se citar: Dias et al. (2012), Mayer et al. (2012) e Lawrence e Balsley (2013). Mais recentemente tem surgido interesse pelo uso de VANTs com rotor, conhecidos como $n$-cópteros, onde $n$ é o número de rotores e os mais comuns são $n=4$ (quadricópteros) e $n=6$ (hexacóptero). Porém, aplicações em meteorologia são quase inexistentes e somente muito recentemente foi reportada em Palomaki et al. (2017). Como vantagens do hexacóptero, como o utilizado aqui, pode-se citar a grande versatilidade que a decolagem vertical propicia e a habilidade para amostrar pontos fixos. Esta habilidade confere ao hexacóptero uma capacidade ímpar para aquisição de perfis verticais. Com a expansão da energia eólica, grande interesse tem surgido por novas técnicas de medidas de vento por instrumentos remotos. Neste trabalho será apresentado um experimento realizado com este objetivo. Os dados gerados neste experimento vêm a preencher uma lacuna de dados fundamentais para a validação de modelos como os utilizados por Paiva et al. (2004) e Pellegrini e Bodstein (2005) que detectaram a necessidade de dados para validarem seus modelos.

\section{2. Área de Estudo e Desenho Amostral}

A região de estudo compreende o reservatório hidrelétrico de furnas situado no interior do estado de Minas Gerais (Fig. 1). A barragem da Usina Hidrelétrica de Furnas está localizada no curso médio do rio Grande, no trecho denominado "Corredeiras das Furnas", entre os municípios de São José da Barra e São João Batista do Glória, em Minas Gerais. A represa cobre uma superfície de $1440 \mathrm{~km}^{2}$ com a cota máxima de $762 \mathrm{~m}$ em relação ao nível do mare abrange 34 municípios de Minas Gerais. O lago é formado por dois braços: um a leste e outro a sul da barragem. Do lado leste, o principal rio que deságua no lago é o Rio Grande. Do lado sul, a represa é formada da junção dos rios Verde, Sapucaí e Machado, além de muitos ribeirões e córregos. O clima predominante é o tropical de altitude, caracterizado por ser mesotérmico, úmido e com chuvas torrenciais. Apresenta temperaturas amenas com poucas variações, além de chuvas no verão e seca no inverno, com variações térmicas entre 21 e $23^{\circ} \mathrm{C}$, e máximas entre 28 e $30^{\circ} \mathrm{C}$. Diferentes projetos geraram dados complementares em diferentes períodos. Dados de 

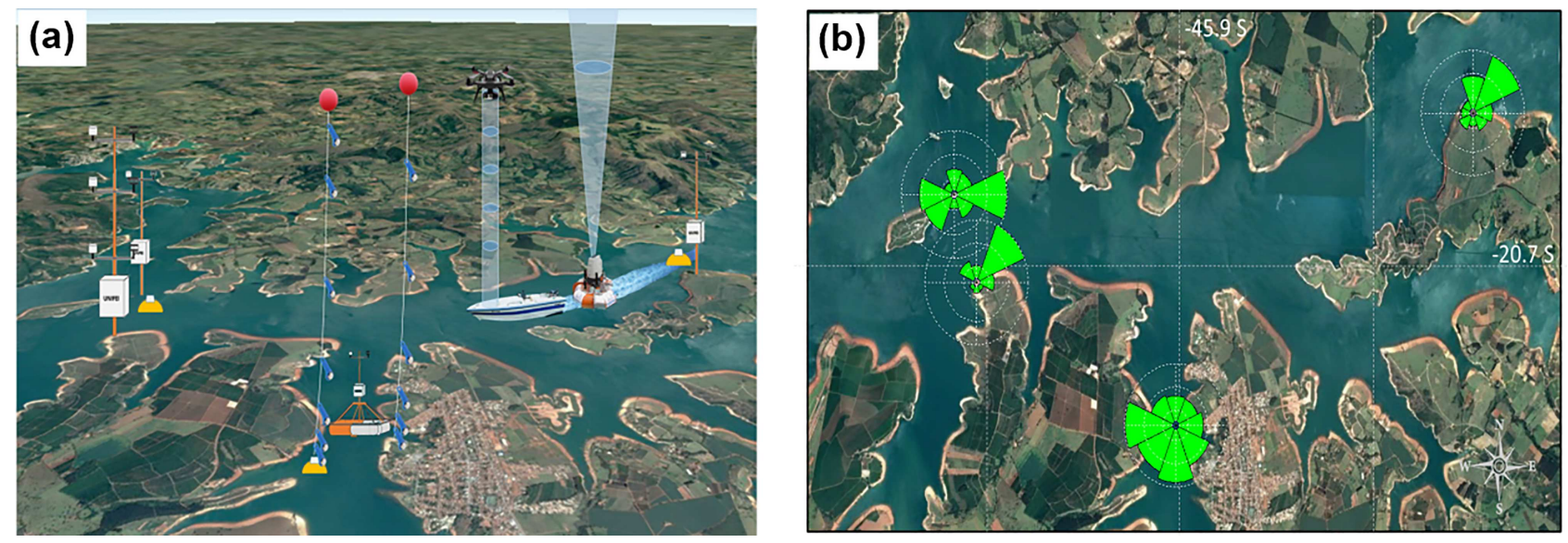

Figura 1 - Área de estudo indicando o arranjo amostral e instrumentações utilizadas (a) e as direções predominantes do vento em quatro locais de medidas (b).

intensidade e direção do vento, temperatura, radiação solar e umidade relativa são provenientes de um experimento que consistiu da instalação de três torres anemométricas num extenso sistema aquático continental, a Usina Hidroelétrica de Furnas em Minas Gerais (Fig. 1a). As torres foram instaladas num arranjo amostral que possibilitou diferentes estudos, dentre eles a investigação do comportamento do escoamento na transição água-terra. Os dados da torre de $42 \mathrm{~m}$ cobriram o período entre novembro de 2013 e setembro de 2014, enquanto as de $10 \mathrm{~m}$ continuam operando até a presente data. Mais recentemente, em campanhas realizadas entre 25 e 30 de setembro e entre 21 e 25 de novembro de 2016 foram realizados experimentos que envolveram vários instrumentos. $\mathrm{O}$ emprego de um LIDAR de última geração possibilitou a coleta de perfis de vento com até $200 \mathrm{~m}$ de altura, que foram complementados com levantamentos realizados a partir de um VANT. Perfis de temperatura e umidade foram realizados a partir de balóes cativos (Fig. 1a). Os dados resultantes foram empregados para cálculos do número de Richardson. A rosa dos ventos indica que, à exceção do setor menos aberto do corpo d'água (ponto mais meridional na Fig. 1b), o vento é predominantemente do primeiro e segundo quadrantes que coincidem com grandes extensões de água.

\section{Instrumentação}

\subsection{Platafora flutuante para prospecção eólica}

Perfis de intensidade e direção do vento são informações fundamentais para estudos meteorológicos, além de serem fundamentais em etapas de prospecção eólica. As radio-sondagens por balões meteorológicos são ainda a fonte principal, a nível global, de perfis do vento (WMO, 2014). Porém, o lançamento operacional e contínuo destas radiossondas ocorre predominantemente em ambientes terrestres sendo esparsas ou inexistentes sobre oceanos e grandes sistemas aquáticos continentais. A prospecção do potencial eólico é uma etapa crítica para um empreendimento na área de energia. Este setor tem, tradicionalmente, se baseado em dados gerados por torres meteorológicas. Enquanto a instalação destas torres é relativamente fácil em ambientes terrestres, nos ambientes aquáticos os custos e logística são quase impeditivos. Torres instaladas em ambiente offshore têm custo entre U\$ 2,5 milhões em áreas não profundas $(<30 \mathrm{~m})$ e atingem valores como U\$ 10 milhões se instaladas a $30 \mathrm{~m}$ de profundidade (Wissemann, 2009). Além do custo, as torres fixas não permitem o fácil deslocamento destas para diferentes setores do sistema aquático. Vários trabalhos têm sido desenvolvidos no sentido do levantamento do potencial eólico em reservatórios hidrelétricos no Brasil (Assireu et al., 2011; Assireu et al., 2013; Pimenta e Assireu, 2015; Assireu et al., 2016). Como a profundidade média destes reservatórios gira em torno de $25 \mathrm{~m}$, e dada a extensão considerável de suas áreas, as questões levantadas acima para o aproveitamento offshore se aplicam também aqui.

À medida que cresce o interesse pelo aproveitamento eólico em sistemas aquáticos, cresce também a necessidade por tecnologias que permitam o dimensionamento do potencial eólico nestes ambientes. O centro Nacional de Dados de Bóias dos Estados Unidos opera uma rede de dados de anemômetros montados sobre bóias. Estas bóias, entretanto, medem ventos entre 3 e $4 \mathrm{~m}$ de altura, o que, somado ao fato destas medidas poderem estar contaminadas pelo movimento da torre em função das ondas, torna estes dados não validados para fins de prospecção eólica (Musial e Ram, 2010). Neste artigo os autores chamam a atenção para a necessidade do desenvolvimento de soluções móveis que permitam determinar o perfil vertical do vento até a altura típica dos aerogeradores modernos.

Em sistemas aquáticos, instrumentos como LIDAR montados sobre navios ou flutuantes têm se apresentado 
como alternativa a radiosondagem e a torres anemométricas por oferecerem perfis com alta resolução temporal (Tucker et al., 2009; Baker et al., 2014). A acurácia dos sistemas LIDAR em relação a torres tem sido verificada tanto para ambientes continentais quanto offshore. A comparação de medidas do LIDAR com medidas em três torres de $63 \mathrm{~m}$ na Dinamarca apontaram para elevados níveis de convergência $\left(\mathrm{R}^{2}=0,97-0,98\right)$ (Hasanger et al., 2011). Comparações com torres de $80 \mathrm{~m}$ também mostraram elevada convergência $\left(R^{2}=0,97\right)$ e viés entre 0,12 e $0,15 \mathrm{~m} / \mathrm{s}$ (Lange, 2011). Kindler et al. (2007) validaram o LIDAR tanto em ambientes terrestres quanto aquáticos. Achtert et al. (2015) compararam as medidas de um LIDAR acomodado sob uma plataforma com compensação das oscilações da superfície com medidas de radiossondagem e verificou viés de $0,3 \mathrm{~ms}^{-1}$ e $2^{\circ}$ e desvio padrão de $1,1 \mathrm{~ms}^{-1} \mathrm{e} 12^{\circ}$ para a intensidade e direção do vento, respectivamente. Todos estes resultados mostram que o LIDAR produz resultados praticamente idênticos àqueles obtidos a partir das tradicionais torres meteorológicas. Como o LIDAR depende de medidas acuradas do desvio Doppler, grandes desafios surgem, relacionados à acurácia, quando estes instrumentos estão a bordo de navios ou flutuantes. Os movimentos destas plataformas, tanto o deslocamento horizontal quanto os movimentos de alta frequência associados às ondas podem induzir erros substanciais nas medidas (Pichugina et al., 2012). Várias pesquisas têm buscado minimizar os erros advindos do movimento das plataformas flutuantes através de mecanismos de compensação. Isto vem sendo realizado basicamente de duas formas: pela medição do movimento da plataforma flutuante (navio, balsa, bóia) e correção no processamento dos dados ou através de mecanismos ativos que estabilizam o LIDAR frente aos movimentos. Dentro desta perspectiva, pelo menos quatro sistemas estão disponíveis comercialmente: SeaZephIR ${ }^{\mathrm{TM}}$, WindSentinel $^{\mathrm{TM}}$, WaveScan ${ }^{\mathrm{TM}}$, andFLiDAR ${ }^{\mathrm{TM}}$. O primeiro sistema limita o movimento do sensor através de mecanismos de compensação e de técnicas de fundeio de forma a tornar desnecessário algorítmos de correção ou de estabilização ativa. Os demais se baseiam em sistemas com meios de estabilização ativa ou correção em nível de processamento. Um exemplo de sistema ativo típico consiste da combinação de dados de GPS para registro dos movimentos de translação combinados a dados de acelerômetros e giroscópio integrados no tempo para determinar a orientação e movimento do LIDAR em tempo real. Esta informação é usada para compensar ativamente a orientação da unidade ótica do LIDAR (Hill et al., 2007).

Neste contexto, nosso grupo direcionou esforços para construir um sistema para prospecção de potencial eólico em ambientes aquáticos (oceano, grandes rios, lagos e reservatórios) baseado em uma balsa ancorada acoplado a sistema de compensação de oscilações, que pudesse acomodar tanto uma torre (Fig. 2a) quanto um LIDAR (Fig. 2b).

\subsection{Balões cativos para levantamento do perfil vertical do vento}

Perfis verticais de temperatura e umidade relativa foram adquiridos a partir do termo-higrômetro portátil do tipo ibutton, marca Embedded data Systems, modelo DS1923 , com $0,6 \%$ de acurácia na umidade relativa e $0,5^{\circ} \mathrm{C}$ de resolução e acurácia na temperatura (Fig. 3a). Estes
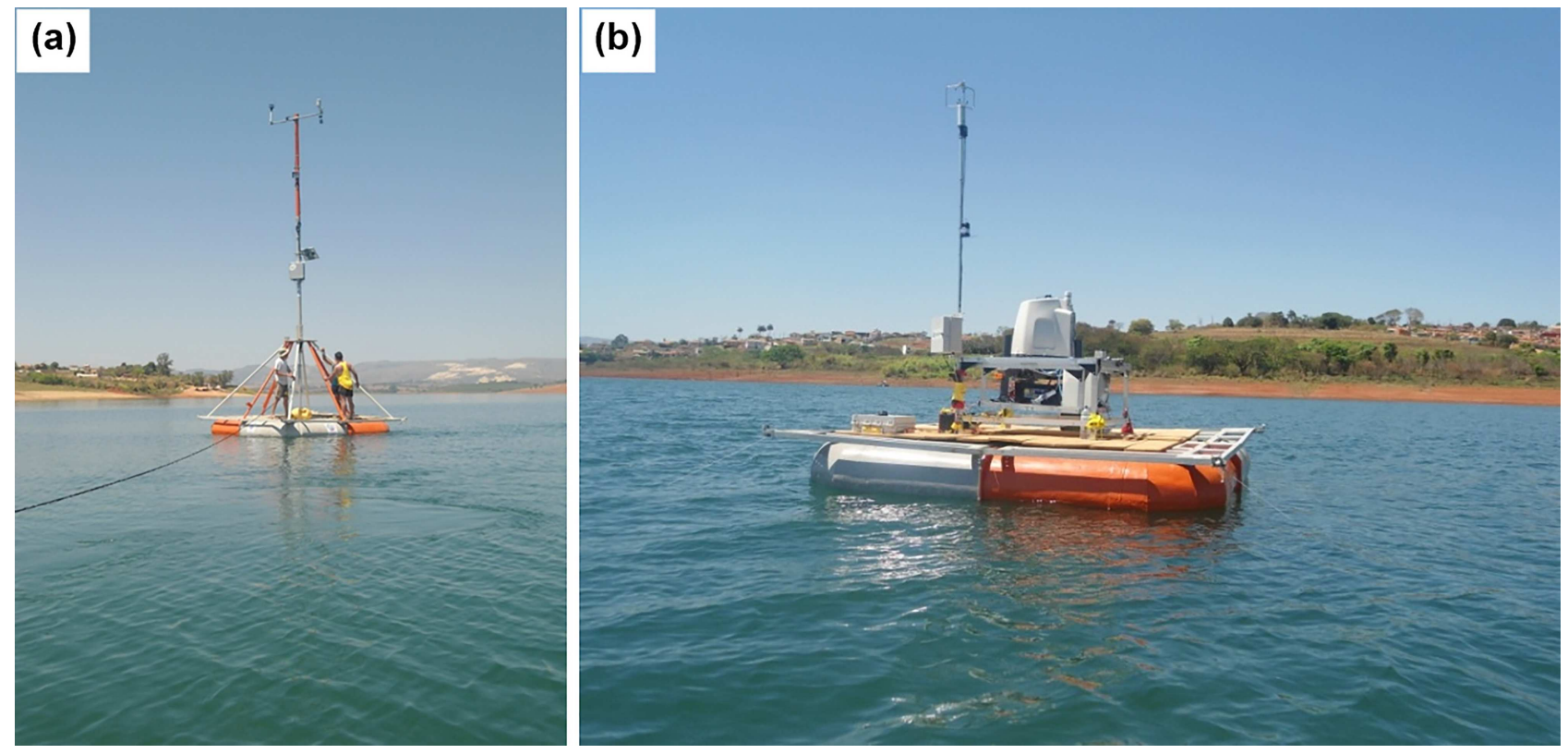

Figura 2 - Sistema móvel para prospecção eólica em sistemas aquáticos hospedando uma torre anemométrica para medidas a $10 \mathrm{~m}$ da superfície (a) e Sistema móvel para prospecção eólica em sistemas aquáticos hospedando um LIDAR (b). 
sensores foram fixados a 1, 3, 5, 7, 10, 20,30, 50 e $90 \mathrm{~m}$ de altura ao longo da corda do balão (Fig. 3b). Foi experimentado também a possibilidade de uso do balão para suportar um anemômetro 3-D juntamente com fonte de energia e dataloger, o qual foi içado a $100 \mathrm{~m}$ de altura. Os perfis de temperatura e umidade, juntamente com medidas de temperatura da superfície da água foram utilizados para estimativas do Número de Richardson, tanto gradiente quanto bulk, para análises das condições atmosféricas quanto à estabilidade.

\subsection{Uso do VANT para prospecção eólica}

O perfil do vento e a intensidade da turbulência são estimados por modelagem numérica, e medidas são imprescindíveis para a validação já que algumas considerações feitas nestes modelos, não necessariamente, são válidas em sistemas aquáticos (Lange et al., 2004). Também, parques eólicos com grande número de aerogeradores são de difícil descrição via modelagem numérica devido às interações entre as esteiras dos diferentes aerogeradores. Assim, medições são fundamentais para melhorar a compreensão destes efeitos. Estas medições podem ser adquiridas por torres meteorológicas ou anemométricas, raras ou inexistentes em sistemas aquáticos brasileiros. Uma alternativa que vem se apresentando viável é o uso de veículos aéreos não tripulados (VANTs) equipados com instrumentação adequada para fazer estas medidas. Esta seção traz os resultados preliminares obtidos a partir de um VANT adaptado para esta finalidade.

\section{Metodologia de Trabalho}

O uso de um hexacóptero (Fig. 4) ao invés dos mais comuns quadricópteros é devido a maior estabilidade do primeiro em relação ao último. Os seis motores trabalham em sincronia de forma a compensar eventuais perturbações causadas por turbulência. O controle de altitude é feito por um barômetro e os motores operam de forma a

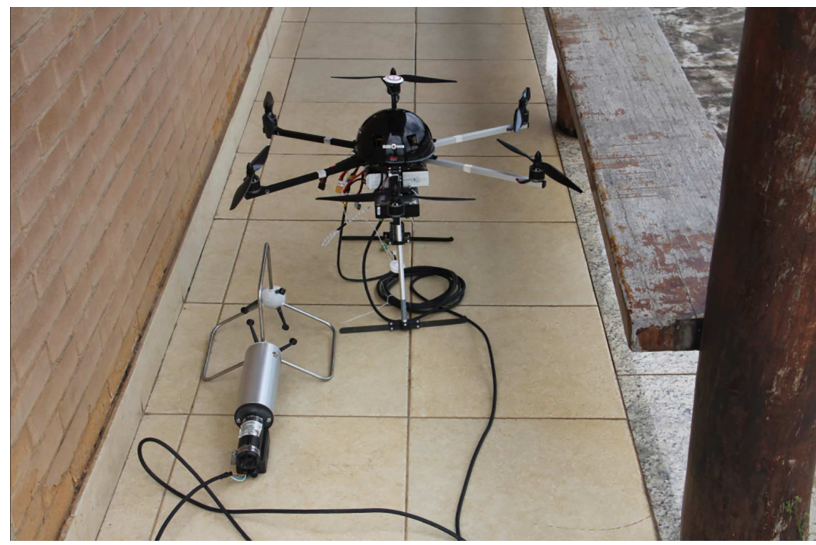

Figura 4 - Hexacóptero utilizado neste estudo e o anemômetro 3-D.

manter a pressão e consequentemente a altitude constante. A Tabela 1 traz as especificações do hexacóptero utilizado.

$\mathrm{O}$ anemômetro utilizado e hospedado como carga útil no VANT foi um modelo que mede além das componentes horizontais também a componente vertical. Tratase de um anemômetro sônico da Young modelo 8100 (Fig. 4). Um datalogger modelo CR1000 da Campbell, instalado próximo às baterias do VANT, foi utilizado para o armazenamento dos dados medidos. A Tabela 2 traz as especificações dos dois tipos de anemômetros utilizados.

$\mathrm{O}$ anemômetro foi preso pelo cabo elétrico de conexão a $4 \mathrm{~m}$ do VANT (Fig. 5). Esta distância foi

Tabela 1 - Especificações do drone utilizado neste trabalho.

\begin{tabular}{lc}
\hline \multicolumn{2}{c}{ Especificações do drone utilizado } \\
\hline Tipo & Hexacóptero X700 \\
Dimensões & $1057 \times 723 \times 462 \mathrm{~mm}$ \\
Distância do motor & $361.5 \mathrm{~mm}$ \\
Peso & $1750 \mathrm{~g}$ (com bateria) \\
Autonomia & $20 \mathrm{a} 25$ minutos \\
Tempo de recarga da bateria & 120 minutos \\
\hline
\end{tabular}
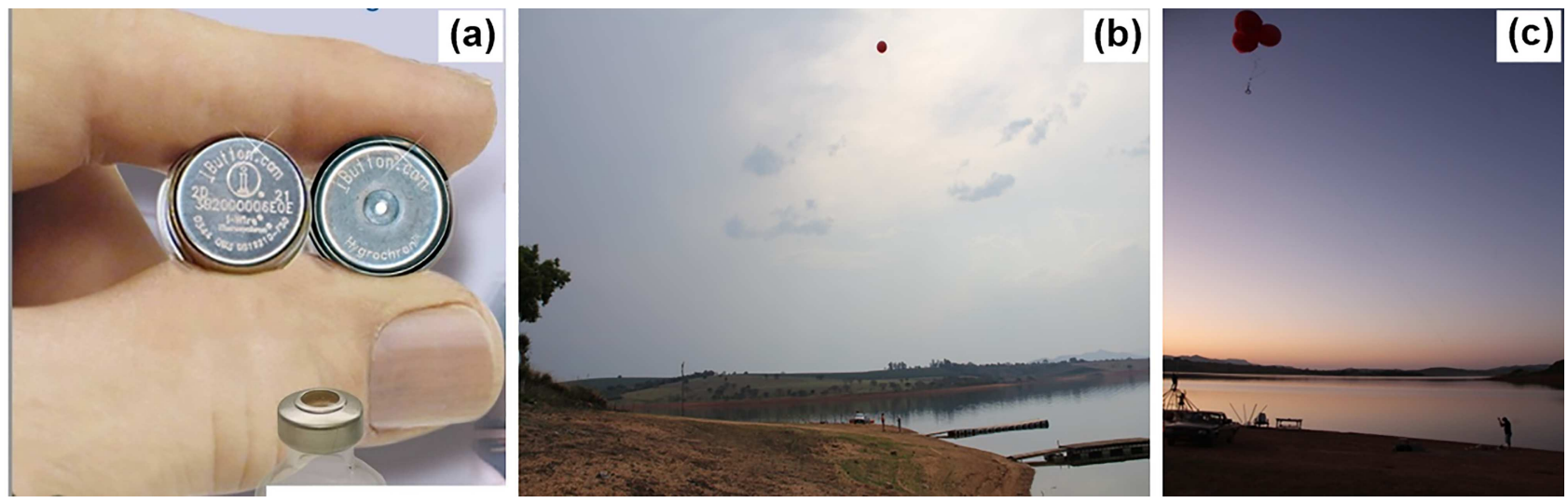

Figura 3 - Sensores de temperatura e umidade compactos (a); perfilamento da temperatura e umidade até $100 \mathrm{~m}$ com o balão (b); uso de balão para medidas de vento a $100 \mathrm{~m}$ de altura (c). 
Tabela 2 - Especificações dos anemômetros utilizados neste trabalho.

\begin{tabular}{lcc}
\hline & Young 3D 8100 & $\begin{array}{c}\text { LambrechtModel } \\
14564\end{array}$ \\
\hline $\begin{array}{l}\text { Intervalo de intensidade } \\
\text { do vento }\end{array}$ & 0 a $40 \mathrm{~m} / \mathrm{s}$ & 0,7 a $50 \mathrm{~m} / \mathrm{s}$ \\
$\begin{array}{l}\text { Resolução da intensidade } \\
\text { Intervalo da direção do } \\
\text { vento }\end{array}$ & $0,01 \mathrm{~m} / \mathrm{s}$ & $0,26 \mathrm{~m} / \mathrm{s}$ \\
$\begin{array}{l}\text { Resolução da direção } \\
\text { Peso }\end{array}$ & 0,1 graus & - \\
Tipo & $1,7 \mathrm{~kg}$ & - \\
& Anemômetro & $0,9 \mathrm{~kg}$ \\
& ultrasônico & Copo
\end{tabular}

empiricamente obtida como a distância a partir da qual a esteira dos motores não influencia as medidas do anemômetro. O relógio do sistema VANT e do datalogger da torre foram sincronizados e o intervalo amostral foi uma medida a cada segundo. Isto possibilitou medidas simultâneas em alturas correspondentes à dos anemômetros da torre de $50 \mathrm{~m}$ da Universidade Estadual do Norte Fluminense (UENF).

\section{Resultados e Discussão}

No dia 28 de outubro de 2016 foi realizado, no campus da Universidade Estadual do Norte Fluminense (UENF), um experimento tendo em vista verificar a viabilidade do uso de um VANT adaptado para fazer perfil vertical do vento. A UENF possui uma torre anemométrica com medidas a 3,10,15, 30 e $50 \mathrm{~m}$ de altura que foram usados como verdade de campo para o experimento. $\mathrm{O}$ anemômetro do VANT foi programado para aquisições em alta frequência $(1,0 \mathrm{~Hz})$, enquanto os valores da torre são expressos em valores médios, mínimos e máximos por minuto, para taxa amostral de $1 \mathrm{~Hz}$. O resultado apresentado na Fig. 6 diz respeito à comparação entre medidas realizadas pelo VANT para as respectivas alturas dos sensores da torre, onde é apresentado o valor médio para cada minuto e o correspondente intervalo de variação das medidas. Os resultados indicam que os valores medidos pelo VANT convergem para o intervalo medido em cada nível pelos respectivos anemômetros e que o perfil obtido por ambas as abordagens também são semelhantes. Os resultados permitiram verificar que para os extremos de vento observados durante o experimento, os quais chegaram a $8,0 \mathrm{~m} / \mathrm{s}$ a $50 \mathrm{~m}$ de altura, o VANT utilizado mostrou-se perfeitamente estável e controlável. Outro resultado importante do experimento foi a verificação de que a autonomia de vôo do VANT utilizado ficou bastante comprometida devido ao peso do conjunto datalogger e anemômetro 3-D. Somente este último pesa próximo a $1,7 \mathrm{~kg}$. Assim, a fim de dar maior capacidade operacional, foi adaptado um anemômetro tipo copo, que pesa metade do peso do utilizado neste experimento, e que deu maior alcance de aplicação ao VANT.

A partir da Fig. 6a nota-se que as medidas realizadas pelo VANT, embora dentro do intervalo de variação das respectivas medidas dos anemômetros da torre, são sempre maiores. A seguinte análise foi realizada tendo em vista investigar tais diferenças observadas. A exceção do anemômetro a $50 \mathrm{~m}$, que é do tipo sônico 2-D da Gill, os demais que equipam a torre são do tipo maritimizado (tipo hélice) da marca Young. Uma comparação entre medidas realizadas em alturas idênticas e no mesmo intervalo de tempo por um anemômetro maritimizado da Young (do mesmo modelo instalado nas torres) e com o hospedado no VANT (3-D sônico da marca Young), mostram que o sônico 3-D tendeu a registrar valores maiores (Fig. 7). Estes dois anemômetros estiveram separados por uma distância próxima a $800 \mathrm{~m}$ e ambos sobre a superfície da água o que tende a diminuir a heterogeneidade espacial do vento. Se esta última afirmativa estiver certa, ou seja, se pudermos assumir que não houve variação espacial substancial do vento na escala de $800 \mathrm{~m}$, então pode-se
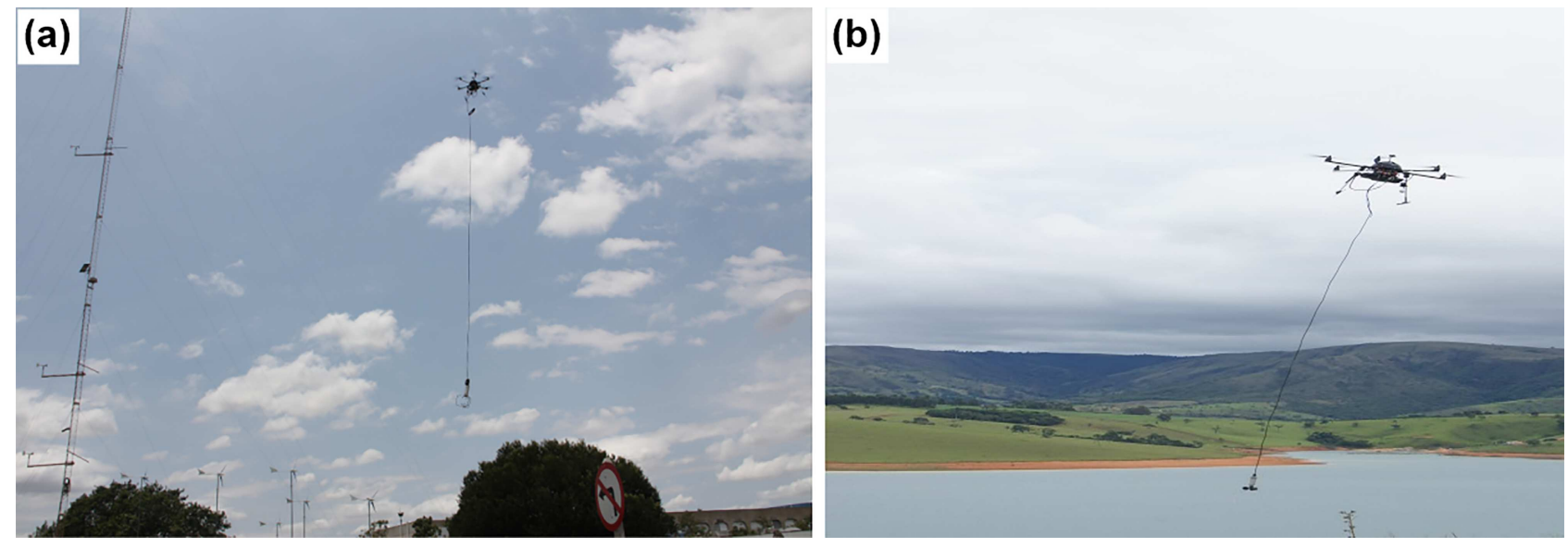

Figura 5 - Perfilamento do vento a partir do VANT próximo a torre anemométrica para fins de comparação (a). VANT em operação no reservatório de Furnas (b). 

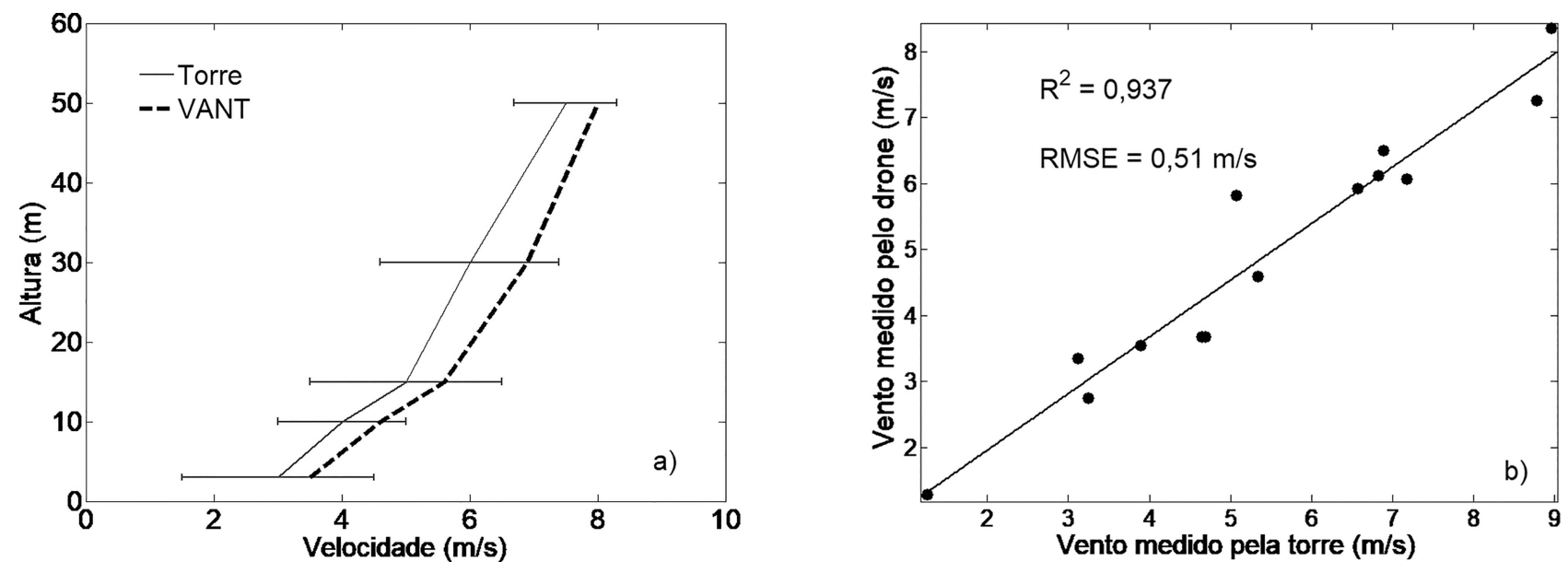

Figura 6 - Perfil do vento levantados pela torre anemométrica e pelo VANT. As barras horizontais indicam a variação do vento no intervalo de 1 minuto no período analisado (a) e comparação dos valores medidos para o anemômetro tipo copo (b).

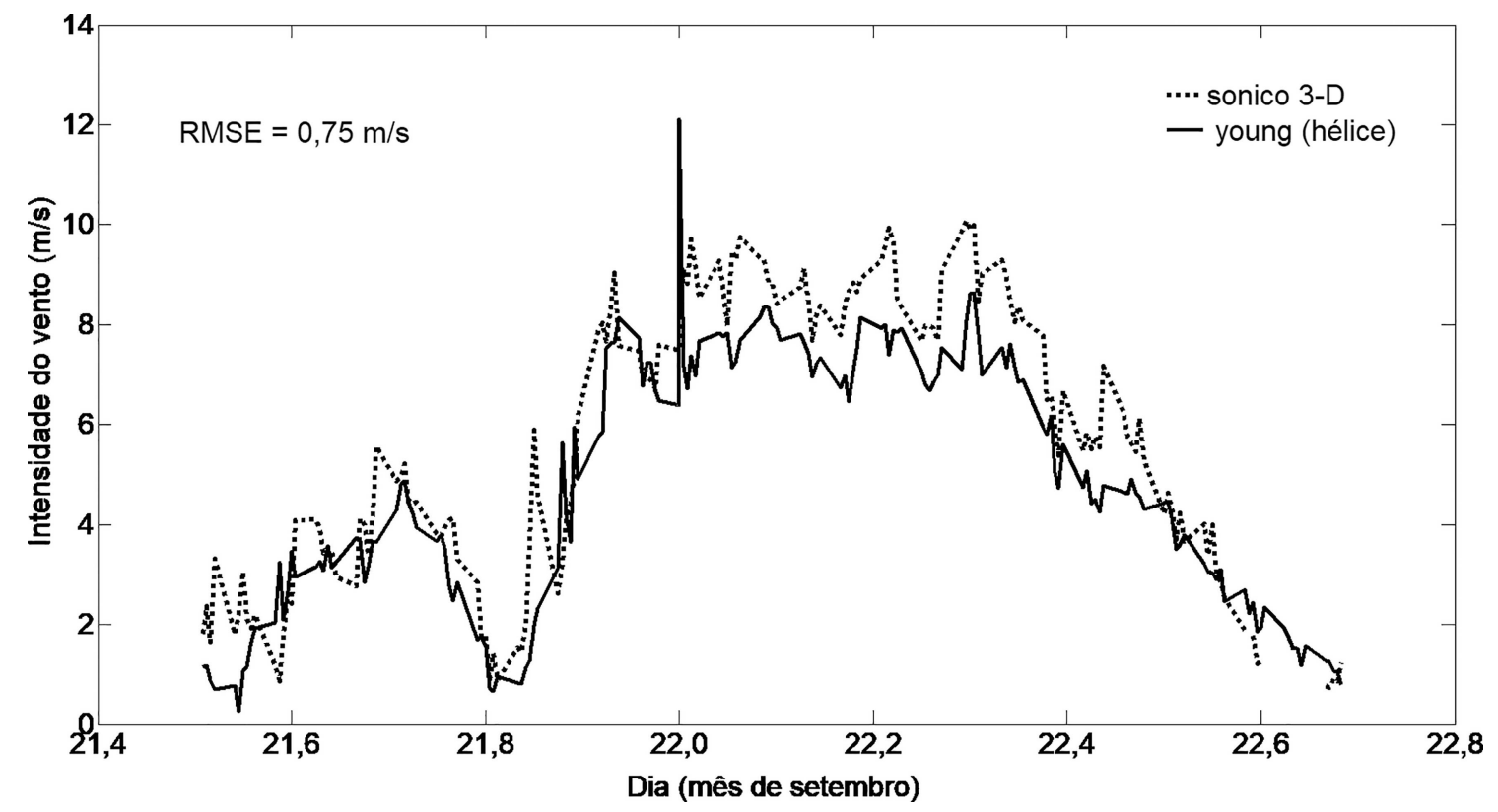

Figura 7 - Comparação do vento medido pelo anemômetro sônico 3-D e pelo tipo maritimizado de hélice, ambos da marca Young.

concluir que os valores maiores registrados pelo VANT são devidos a uma ligeira super-estimativa do sônico 3-D quando comparado ao maritimizado da Young. Um experimento especifico, que instale estes dois anemômetros próximos, deverá ser realizado para informações mais acuradas acerca desta questão.

\section{Conclusões}

Experimentos intensivos multi-instrumentos realizados num dos maiores reservatórios do Brasil, resultaram em dados que permitirão avanços importantes no conhecimento da camada limite atmosférica (CLA) influenciada por extensos sistemas aquáticos continentais marcados por orografia complexa. Os dados foram coletados predominantemente sob condições de atmosfera instável, mas cobriram também períodos de condições estáveis e neutra. Os resultados obtidos vêm a preencher uma lacuna importante de dados relativos à CLA em regiões de terreno complexo e de transição entre terra e água. Este artigo visou apresentar informações gerais sobre $o$ desenho amostral e instrumentações que geraram dados que subsidiarão uma série de artigos científicos sobre resultados dos projetos realizados. Isto inclui detalhes sobre a resposta do perfil do vento ao fetch, colinas e influências do terreno e mudança abrupta de rugosidade na interface água-terra.

Neste artigo visou-se também apresentar o resultado de um avanço metodológico e instrumental cujos dados serão amplamente utilizados nos artigos comentados 
acima. No final do mês de outubro de 2016 foi concebido e realizado um experimento, no campus da Universidade Estadual do Norte Fluminense (UENF), que visou investigar o uso de um hexacóptero para sustentar um anemômetro tridimensional, para fins de perfilhamento do vento. Os dados de uma torre anemométrica com medidas a 3, 10, 15,30 e $50 \mathrm{~m}$ foram utilizados como verdade de campo. Os experimentos foram realizados em condições de ventos moderadamente intensos, valores atingiram $8,0 \mathrm{~m} / \mathrm{s}$ a $50 \mathrm{~m}$ e em condição de atmosfera instável. Sob estas condições o VANT mostrou-se bastante estável e controlável. Comparações entre as medidas feias pelo VANT e a torre em alturas correspondentes indicou convergência dos dados. Uma limitação observada foi a redução drástica na autonomia de vôo o que esteve associado ao elevado peso do anemômetro utilizado, cerca de $1,7 \mathrm{~kg}$ - fora o datalogger e cabo elétrico. Isto impulsionou o uso de um anemômetro de copo, muito mais leve. Resolvida a questão da autonomia, foram realizadas campanhas com o VANT na área de estudo o que permitiu levantar perfis com cobertura espacial e a alturas ainda não realizadas em sistemas aquáticos brasileiros. Os resultados que serão tratados nos outros artigos, baseados nestes dados, trarão contribuições importantes e inéditas sobre o comportamento da CLA sobre colinas e influenciadas pelo fetch e detalhes topográficos e de cobertura superficial que influenciam o escoamento. A conclusão final é que o VANT adaptado para medidas de parâmetros atmosféricos representa uma promissora plataforma para o perfilamento vertical da CLA.

\section{Agradecimentos}

À FAPEMIG (Projeto APQ 1575/14). ATA e FMP agradecem o apoio financeiro do $\mathrm{CNPq}$, respectivamente, pelos projetos CNPq (309315/2015-8) e CNPq (406801/2013-4, 465672/2014-0, 311930/2016-6). À Equinócio Geoambiental pelo fornecimento e suporte ao uso do VANT. Os autores agradecem aos dois revisores pelas importantes sugestões e correções.

\section{Referências}

ACHTERT, P.; BROOKS, B.J.; MOAT, B.I.; PRYTHERCH, J.; PERSSON, P.O.G.; TJERNSTRÖM, M. Measurement of wind profiles by motion-stabilised ship-borne Doppler lidar. Atmospheric Measurement Techniques, v. 8, n. 11, p. 4993-5007, 2015.

ASSIREU, A.T.; MENDONÇA, J.C.; PASSOS, R.B.; PELLEGRINI, C.C.; FREITAS, R.M. Escoamento atmosférico sobre uma colina isolada revelado a partir de dados de um veículo aéreo não tripulado (VANT). Revista Brasileira de Meteorologia, v. 34, n. 2, 227-236, 2019.

ASSIREU, A.T.; PIMENTA, F.; SOUZA, V. Assessment of the wind power potential of hydroelectric reservoir. In: EnnerAlcântara. (Org.). Energy Resources: Development,
Distribution, and Exploitation. 1ed. Nova York: Nova Science Publishers, p. 1-30, 2011.

ASSIREU, A.T.; PELLEGRINI, C.C.; Pimenta, F.M. Intensificação do Vento Devido a Influências do Relevo: Evidências a Partir de Modelos Numéricos e Medidas In Situ. Ciência e Natura, v. Especial, p. 60-62, 2013.

ASSIREU, A.T.; PIMENTA, F.M.; FREITAS, R.M. Observação e modelagem da camada limite atmosférica na interface água-terra. Ciência e Natura, v. 38, p. 305, 2016.

ASSIREU, A.T.; TIAGO FILHO, G.L.; FREITAS, R.M.; MENDONCA, J.C.; PIMENTA, F.M. Sistema flutuante móvel para prospecção de potencial eólico em sistemas aquáticos. 2012, Brasil. Patente: Privilégio de Inovação. Número do registro: BR1020120054876, título: "Sistema flutuante móvel para prospecção de potencial eólico em sistemas aquáticos", Instituição de registro: INPI - Instituto Nacional da Propriedade Industrial. Depósito: 12/03/2012.

BAKER, W.E.; ATLAS, R.; CARDINALI, C.; CLEMENT, A.; EMMITT, G.D.; GENTRY, B.M.; HARDESTY, R.M.; KÄLLÉN, E.; KAVAYA, M.J.; LANGLAND, R.; MA, Z.; MASUTAMI, M.; MCCARTY, W.; PIERCE, R.B.; PU, Z.; RIISHOJGAARD, L.P.; RYAN, J.; TUCKER, S.; WEISSMANN, M.; YOE, J.G. Lidar-measured wind profiles: the missing link in the global observing system. Bulletin of the American Meteorological Society, v. 95, n. 4, p. 543-564, 2014.

BANGE, J.; BEYRICH, F.; ENGELBART, D.A.M. Airborne Measurements of Turbulent Fluxes during LITFASS-98: A Case Study about Method and Significance. Theoretical and Applied Climatology, v. 73, n. 1, p. 35-51, 2002.

BEYRICH, F.; MENGELKAMP, H.-T. Evaporation over a Heterogeneous Land Surface: EVA GRIPS and the LITFASS2003 Experiment - an Overview. Bounday-Layer Meteorology, v. 121, n. 1, p. 1-28, 2006.

DIAS, N.L.; GONÇALVES, J.E.; FREIRE, L.S.; HAZEGAWA, T.; MALHEIROS, A.L. Obtaining potential virtual temperature profiles, entrainment fluxs, and spectra from mini unmanned aerial vehicle data. Boundary-Layer Meteorology, v. 145 n.1, p. 93-111, 2012.

HILL, R.; BREWER, W.A.; TUCKER, S. Platform-motion correction of velocity Measured by Doppler Lidar. Journal of Atmospheric and Oceanic Technology, v. 25, n. 8, p. 1369-1382, 2008.

HASANGER, C.; BADGER, M.; PEÑA, A.; BADGER, J.; ANTONIOU, I.; NIELSEN, M.; ASTRUP, P.; COURTNEY, M.; MIKKELSEN, T. Advances in offshore wind resource estimation. In Advances in Wind Energy Conversion Technology, Matthew S, Philip G (eds), p. 85-106, 2011.

KINDLER, D.; OLDROYD, A.; MACASKILL, A.; FINCH D. An eight month test campaign of the QinetiqZephIR system: Preliminary results. MeteorologischeZeitschrift, v. 16, n. 5, p. 479-489, 2007.

LANG, S.; MCKEOGH, E. LIDAR and SODAR measurements of wind speed and direction in upland terrain for wind energy purposes. Remote Sensing, v. 3, n. 9, p. 1871-1901, 2011.

LANGE, B.S.; LARSEN, J.; HOJSTRUP, R.; ARTHELMIE, R. Importance of thermal effects and the sea surface 
roughness for offshore wind resource assessment. Journal of Wind Engineering and Industrial Aerodynamics. v. 92, n. 11, p. 959-988, 2004.

LAWRENCE, D.A.; BALSLEY, B.B. High-resolution atmospheric sensing of multiple atmospheric variables using the DataHawk small airbone measurement system. Journal of Atmospheric and Oceanic Technology, v. 30, n. 10, p. 2352-2366, 2013.

NASSIF, F.B.; PIMENTA, F.M.; ASSIREU, A.T. Uso do LIDAR para o levantamento do perfil do vento em extensos sistemas aquáticos: Primeiros resultados. Revista Brasileira de Meteorologia, submetido.

MAYER, S.; SANDVIK, A.; JONASSEN, M.; REUDER, J. Atmospheric profiling with the UAS SUMO: a new perspective for the evaluation of fine-scale atmospheric models. Meteorology and Atmospheric Physics, v. 116, n. 1-2, p. 15-26, 2012.

MUSIAL, W.; RAM, B. Large-scale offshore wind power in the United States: Assessment of opportunities and barriers. National Renewable Energy Laboratory Technical Report. NREL/TP-500-40745, 221 pp., 2010.

PALOMAKI, R.T.; NATHAN, T.R.; BOSSHE, M.V.D.; SHERMAN, T.J.; WEKKER, S.F.J. Wind estimation in the lower atmospheric using multirotor aircraft. Journal of Atmospheric and Oceanic Technology, v. 34, n. 5, p. 11831191, 2017.

PAIVA, L.M.S.; BODSTEIN, G.C.R.; MENEZES, W.F.; Simulação do escoamento atmosférico sobre uma colina isolada utilizando o modelo RAMS. Revista Brasileira de Meteorologia, v.19, n.2, p. 163-176, 2004.

PELLEGRINI, C.C.; PIMENTA, F.M.; ASSIREU, A.T. O desempenho do WRF num episódio de intenso e persistente vento num grande reservatório tropical. Revista Brasileira de Meteorologia, v. 34, n. 1, p. 121-138, 2019.

PICHUGINA, Y.; BANTA, R.; BREWER, W.; SANDBERG, S.; HARDESTY, R. Doppler lidar-based wind-profile measurement system for offshore wind-energy and other marine boundary layer applications. Journal of Applied Meteorology and Climatology, v. 51, n. 2, p. 327-349, 2012.

PIMENTA, F.M.; ASSIREU, A.T. Simulating reservoir storage for a wind-hydro hydrid system. Renewable Energy, v. 76, p. 757-767, 2015.

REIS, A.L.; PIMENTA, F.M.; ASSIREU, A.T. Uso do LIDAR para o levantamento do perfil do vento em extensos sistemas aquáticos: Primeiros resultados. Revista Brasileira de Meteorologia, submetido.

TUCKER, S.C.; SENFF, C.J.; WEICKMANN, A.M.; BREWER, W.A.; BANTA, R.M.; SANDBERG, S.P.; LAW, D. C.; HARDESTY, R.M. Doppler lidar estimation of mixing height using turbulence, shear, and aerosol profiles. Journal of Atmospheric and Oceanic Technology, v. 26, n. 4, p. 673-688, 2009.

WMO, Statement of guidance for global numeric weather prediction (NWP), available at: www.wmo.int/pages/prog/ www/OSY/GOS-RRR.html (last access: 30 June 2015), 2014.

WISSEMANN, C. IOOS. Offshore Wind energy Meeting presentation. Rutgers University, 2 February 2009.

This is an Open Access article distributed under the terms of the Creative Commons Attribution License, which permits unrestricted use, distribution, and reproduction in any medium, provided the original work is properly cited. 